\title{
COMPLEX NUMERICAL MODELING OF DYNAMICS AND CRASHES OF WING-IN-GROUND VEHICLES
}

\author{
Nikolai Kornev $^{*}$ \\ University of Rostock, Rostock,18059, Germany \\ and \\ Konstantin Matveev ${ }^{\dagger}$ \\ California Institute of Technology, Pasadena, CA, 91125, USA
}

\begin{abstract}
$\underline{\text { Abstract }}$
The Wing-In-Ground craft (WIG), a vehicle flying in the ground effect, is a promising transportation means of the near future. This paper describes mathematical modeling of WIG motion in all regimes, such as planing, take-off, transition to flight, and flight itself. The model, which includes nonlinear hydroaerodynamics, serves as a base for simulation of motion. The theory developed here enhances the process of designing WIG vehicles; its advantages and disadvantages are discussed. The results of numerical modeling are compared with experimental data obtained for planing and flight regimes of motion. The model is applied for studying emergency problems in WIG operation.
\end{abstract}

\section{Introduction}

The Wing-In-Ground craft (WIG), called also ekranoplan and Ground Effect Machine (GEM), is the high-speed low-altitude flying vehicle that utilizes a favourable ground effect. This effect appears at distances from the ground about one wing chord and results in the enhanced lift-drag ratio. As a transportation means, WIG is positioned in the niche between ships and aircraft: speeds of WIG are much higher than those of ships, and operational expenses are much lower than those of airplanes. Another advantage of most WIG vehicles is their amphibious properties; moreover, they can take off and land at any relatively flat surface, such as land, water, snow, and ice. Military WIG craft fly beyond the air defence radars' zone, and are invulnerable to mine-torpedo weapons. The usage of the ground effect is also discovered in nature: birds and flying fish spend less energy moving in the vicinity of water surface. ${ }^{2}$

The most significant contribution to the progress of the WIG concept was made in Russia by the Central Hydrofoil Design Bureau under the guidance of R.E. Alekseev, who developed a number of unique test craft (the series SM and the famous Caspian Monster KM), as well as the first serial vehicles of Orlyonok (Fig. 1) and Lun types. Two generations of ekranoplans were constructed and successfully tested. ${ }^{1}$ Due to a low commercial potential of these craft designed for the Navy and a high cost of the development, large ekranoplans did not find practical application in the new economic and political situation of the last decade. At the present time, more attention is paid to the development of manually controlled small craft (Amphistar, FS8, Hoverwing, Hydrowing, TAF and Chinese craft). ${ }^{2}$ The ground effect, resulting in the considerable increase of the lift and the lift-drag ratio, makes the efficiencies of these WIG vehicles higher than those of the other transportation means in the speed range from $60 \mathrm{mph}$ to $300 \mathrm{mph}$. A recent success of FS8 vehicle, designed by German firm AFD, ${ }^{3}$ raises a hope that WIG craft will find their niche in the transportation system in the near future.

Unique characteristics of WIG make it a suitable platform for various tasks. The usage of WIG is studied for rescue operations in the ocean and as a first stage in the sea launch of reusable aerospace planes. ${ }^{b, c}$ Boeing Phantom Works has recently announced a project on a

\footnotetext{
* Visiting Professor, Institute of Thermodynamics, nikolai.komev@mbst.uni-rostock.de

†Ph.D. candidate, Mechanical Engineering, matveev@caltech.edu
} 
high-capacity cargo plane using a ground effect for military and commercial purposes.

Mathematical methods used for flight regimes of WIG operation can be divided in three groups. Analytical methods, based on conformal mapping and jet theory, allow to find solutions only for very limited class of two-dimensional problems. Asymptotic approaches assume a solution as a series of a small parameter. The method of $\tau$-parameter and the method of matched asymptotic expansions were successfully applied for WIG problems. Numerical methods, based on the potential theory, as well as viscous solvers, can treat realistic geometries; however, most CFD techniques require considerable $\mathrm{CPU}$ time and are not suited for optimization design. A brief review of theoretical and numerical approaches can be found in the book by Rozhdestvensiky.

The successful and effective design of the WIG craft operating in the vicinity of the air-sea interface remains a challenging problem. The main reason for the distrust of customers with regard to WIG, besides its unusualness, is the periodic crashes that occurred with craft build by newcomers, who do not have sufficient financial means and time to gather necessary experience before attempting full-scale flights. Unfortunately, renowned designers have also had a series of accidents, whose reasons are often not completely clear. In this sense, the mathematical modelling of the WIG motion under emergency conditions becomes a problem of great importance. This paper describes an effort to construct a predictive tool aimed at developing a piloting strategy in the critical motion regimes to prevent crashes.

The complex model presented here contains the following sub-models: hydrodynamics of the transitional mode, planing of stepped surfaces, aerodynamics in the ground effect, unsteady hydroaerodynamics, modelling of the wave-wind effects, and the simulation of motion. The hydro-aerodynamic model is based on the Nonlinear Vortex Lattice Method (NVLM). Using this model, the commercial code Autowing has been developed and is in use by several companies manufacturing small WIG craft., ${ }^{4,5}$

\section{Mathematical Model}

The modeling of a wing motion in proximity to the ground includes a large variety of methods ranging from simple channel models, analytical asymptotic approaches, potential panel methods, and modern finite volume methods. ${ }^{6}$ The most critical design problems can be solved by use of panel methods. The vortex lattice method (VLM), used here, represents the best trade-off between accuracy and required computational resources for a certain class of problems. Within the developed theory, the simulation of WIG motion is based on the full nonlinear system of equations describing three dimensional dynamics of WIG craft in all regimes of its motion. The hydro-aerodynamic forces are calculated at every time step.

The motion of a WIG before the take-off can be divided into two regimes:

1) transitional mode, when the Froude number $F r=V_{\infty} / \sqrt{g D^{1 / 3}}<3$, where $V_{\infty}$ is the speed and $D$

is the displacement of a vehicle;

2) planing mode with aerodynamic unloading, when Fr $>3$.

Determination of hydrodynamic forces and parameters of the WIG motion in the first regime is a very difficult theoretical problem, which is even more complicated due to the presence of a stepped bottom. We implement the approach based on the results of model testing in a towing tank. The planing surface is treated as a wing with an unknown wetted surface, which is determined using a special iterative technique that makes the problem nonlinear. To find the wave surface behind the step, a wave theory is applied, enforcing the kinematic and dynamic boundary conditions on the free surface. The free surface is represented via a number of closed vortex frames with intensities found from the free surface dynamic boundary condition. Once the wetted surface of a planing hull is known, it can be considered as a conventional wing. The VLM method can be applied to determine the unsteady derivatives and added masses are calculated at every time moment for the instant (frozen) wetted surface. Every aerodynamic coefficient $C$ is represented as a sum of quasi-steady, unsteady and perturbation parts:

$$
\begin{aligned}
C= & \underbrace{C(\vartheta, h, V)+C^{\beta} \beta+C^{\gamma} \gamma+C^{\Omega_{y}} \Omega_{y}}_{\text {quasi-steady }}+ \\
& +\underbrace{C^{\dot{h}} V_{y} / V+\left[C^{\dot{\gamma}} \Omega_{x}+C^{\dot{\theta}} \Omega_{z}\right] L / V}_{\text {unsteady }}+C_{w w}
\end{aligned}
$$

where $\vartheta, h, \beta, \gamma$ are respectively the pitch angle, the flight height, the drift angle, and the roll angle; $\Omega_{i}$ are the components of the angular velocity, $V$ is the speed of motion with respect to a quiescent air, $C_{w w}$ is responsible for additional forces due to wave and wind perturbations. All coefficients in $C$ are nonlinear functions of $g$ and $h$. These coefficients are calculated using the NVLM method. The nonlinear boundary conditions are satisfied on the actual positions of lifting surfaces at every time step. The viscous drag is calculated employing empirical formulae. The additional forces $C_{w w}$ are found from the special model proposed for the case of small-amplitude waves. ${ }^{7}$ 


\section{Validation of the model}

The mathematical model was thoroughly tested for motion characteristics in planing and flight modes. Figures 2 and 3 show the drag, lift and moment coefficients for various WIG craft, which compare well with the wind tunnel data.

The current model gives reasonable agreement with the TSAGI measurements for the planing mode. The slope of the lift coefficient is presented in Fig. 3. The shape of the wave wake behind the step, shown in Fig. 4 , compares well with the semi-empiric models of Epstein (dashed line) and Payne ( $\diamond)$.

\section{Numerical investigations of operational safety on a WIG with manual handling}

The goal of this section is to give some recommendations how the designers and owners of small WIG craft (without automatic control system) can estimate safety of operation. All hydro- and aerodynamic characteristics mentioned below can be found using the methods described above.

Estimation of the operational safety of WIG craft in cruise motion

Linear analysis of the static stability. Starting point for estimation of operational safety of WIG craft is the linear analysis of stability in cruise motion, which comprises static and dynamic stability. The most important issue for WIG operation is the static stability. The requirements for the dynamic stability are usually satisfied if the system parameters, which provide the static stability, are within a certain (narrower) range. The criteria of the static stability can be written in the form proposed by Irodov ${ }^{7}$

$$
\frac{\partial C_{m}}{\partial \vartheta}<0
$$

$$
\Delta X>0,
$$

where $\quad \Delta X=X_{h}-X_{\vartheta} ; \quad X_{h}=\frac{\partial C_{m}}{\partial h} / \frac{\partial C_{l}}{\partial h} \quad$ aerodynamic center on altitude (NA); $X_{\vartheta}=\frac{\partial C_{m}}{\partial \vartheta} / \frac{\partial C_{I}}{\partial \vartheta}-$ aerodynamic center on pitch (NP); $C_{m}$ and $C_{l}$ are the pitching moment and lift coefficients respectively; $h$ is the flight altitude, $\vartheta$ is the pitch angle. The positions of aerodynamic centers are measured from the center of gravity $(\mathrm{CG})$. The first condition (1) can be easily satisfied, for instance, by use of tail unit; whereas ensuring the second requirement
(2) requires a certain design skill. The three most important factors influencing the Irodov's criterion (2) are the following: the tail unit, the profiles of wing sections, and the main wing form. In contrast to a conventional aircraft, the position of the center of gravity does not influence the static stability qualitatively. In other words, a statically unstable WIG craft can not become stable by shifting the center of gravity

As experience shows, the motion stability is provided if $\Delta X$ from the criterion of the static stability (2), divided by a mean aerodynamic chord, is within the range $0.05-0.15$. If $\Delta X$ is greater than 0.15 , then the static stability becomes excessive; that can lead to the dynamic instability. Usually this happens at a flight altitude much less than a cruise altitude. If $\Delta X$ is positive but less than 0.05 , then the stability is insufficient; that leads to the long-period (phugoid) instability. This consideration of the static stability can be taken as the principle for selecting the maximum possible altitude of a stable flight for a given pitch angle, as well as for selecting the maximum possible pitch angle of a stable motion for a given flight altitude. The suitable for that purpose diagram is given in the Fig. 6.

The condition $\Delta X>0.05$ is applied to determine the area of the sufficient stability (Fig. 6). The region between the area of sufficient stability and the area of inadmissible flight altitude corresponds to insufficient stability. Information in Fig. 6 can be used as a guideline for safe piloting. The speed of motion related to the chosen height of flight and pitch angle can be found from the simple equilibrium equation $V=\sqrt{2 G /\left(C_{l} \rho S\right)}$, where the lift coefficient $C_{l}$ is calculated by methods described above, and $G$ is the weight of the craft. The pitching moment, if it is non zero, can be compensated by a deflection of the flap on the tail unit. The less the pitch angle, the higher is the boundary of the static stability (Fig. 6). This tendency is violated at small flight altitudes for pitch angles less than -3 degrees. The reason for that is the Ventouri effect.

Favorable position of the center of gravity. Providing that a WIG craft is designed properly, so that its parameters are in the area of sufficient stability, the next question is a choice of the position of the center of gravity $X_{c g}$. As we said above, the position of $\mathrm{CG}$ does not change the stability qualitatively; however, it can affect dynamics of WIG craft and consequently safety of operation.

Sensitivities of the pitch angle and flight altitude to variation of the vehicle velocity are important for determining a favourable position of the gravity center. These dependences can be obtained from the equations 
of WIG equilibrium. ${ }^{7}$ The derivative of the pitch angle on the speed of motion is

$$
\frac{d \vartheta}{d V}=-\frac{2}{V} \frac{C_{l}}{\partial C_{l} / \partial \vartheta} \frac{X_{h}}{X_{h}-X_{\vartheta}}
$$

the derivative of the flight altitude on the speed of motion is

$$
\frac{d h}{d V}=\frac{2}{V} \frac{C_{l}}{\partial C_{l} / \partial h} \frac{X_{\vartheta}}{X_{h}-X_{\vartheta}} .
$$

Analysing Eqs. (1-4), we come to the following conclusions:

1) If the aerodynamic center on altitude coincides with the center of gravity, then $X_{h}=0$, and the pitch angle does not vary with speed. This result follows from the expression (3).

2) If the center of gravity is between aerodynamic centers NA and NP, then $X_{h}>0$ and $\Delta X>0$; and the pitch angle decreases with increasing speed of motion (Eq.3).

3 ) If the center of gravity of a stable WIG craft is between the aerodynamic centers NA and NP, then $X_{g}<0, X_{h}>0$, and $X_{9} /\left(X_{h}-X_{\vartheta}\right)<1$; and the damping effect of the ground is increased (Eq. 4). This is analogous to the increase of derivative $\partial C_{l} / \partial h$.

4) If the center of gravity of a stable WIG craft is in front of the center on altitude, then $\Delta X>0, X_{\vartheta}<0$, $X_{h}<0$, and $X_{\vartheta} /\left(X_{h}-X_{\vartheta}\right)>1$; and the damping effect of the ground is decreased (Eq. 4).

These conclusions lead to formulating the following requirement for a position of the center of gravity (Fig. 7): ${ }^{8}$ the center of gravity should be located between the aerodynamic centers on altitude and pitch and close to the center on altitude:

$$
X_{h}>X_{c g}>X_{\vartheta}, X_{c g} \sim X_{h} .
$$

The conditions (5) allows one to choose a favourable position of the center of gravity using a diagram presented in the Fig. 8. According to requirement (5), the center of gravity should be close to the aerodynamic center in altitude. In this study, we define a favourable position of the center of gravity (CG) as a location between the center on altitude and the middle point between two aerodynamic centers $0.5\left(X_{h}+X_{\vartheta}\right)$.

Linear analysis of dynamic stability. Another limitation on the center of gravity is imposed by requirements of providing dynamic stability. To ensure dynamic stability, CG should be located in a certain range.
Calculations done for the WIG craft Seajet revealed the following facts typical for small WIG vehicles:

1) The range of $\mathrm{CG}$ position that satisfies dynamic stability is within the zone of favorable positions defined above (Eq. 5 and Fig. 8). Therefore, the condition (5) is necessary for dynamic stability.

2) Providing that the margin of static stability is sufficient, there is a range of CG position that ensures dynamic stability. The range of flight altitudes where a WIG craft is dynamically stable is almost the same as the range where the WIG is stable statically. The dynamic stability of a statically stable WIG can be obtained by a proper positioning of CG.

3) If the margin of static stability is small $(\Delta X \sim 0)$, then dynamic stability cannot be provided for any $C G$ position.

When a pilot can regulate only the thrust of a WIG craft, whereas the flight altitude and the pitch angle are not controlled directly, the shifting a CG position is ambiguous from the viewpoint of stability. On one hand, reduction of the pitch angle caused by shifting a CG position towards the leading edge results in the improvement of stability. On the other hand, it leads to an increase of the flight altitude and to the loss of natural stability provided by the ground vicinity. Usually, the second effect appears to be stronger

Simulation of WIG motion. Influence of wind gusts and waves on WIG dynamics at different positions of CG. Simulation of the perturbed motion of the WIG craft considered here show that oscillations of trajectory are minimal and the response to a wind gust is mild when the center of gravity is located between aerodynamic centers. If the center of gravity is located either in front of the center on altitude or behind the center on pitch, then oscillations are stronger and the wind gust response is much harder. We presume that a pilot has means to attain a given (cruise) regime of motion by changing the thrust and deflecting the flap on the tail unit.

The averaged wind speed $W_{a v}$, corresponding to the gust speed $W_{x \max }$ acting on a WIG craft, can be estimated assuming that a deviation of the wind speed near the water surface is about 0.09 of the averaged wind speed: ${ }^{9}$

$$
W_{a v}=W_{x \max } / 0.09 \text {. }
$$

We investigated the flight of the small WIG craft Seajet taking the distance between the fuselage and undisturbed water surface to be $-0.7 \mathrm{~m}$ and the speed to be $\sim 41 \mathrm{~m} / \mathrm{sec}$. Zero pitch angle was attained by deflecting the flap on the tail unit. In this equilibrium regime, the aerodynamic center on altitude was located at the distance $2.10 \mathrm{~m}$ from the leading wing edge, 
whereas the aerodynamic center on pitch was at the distance $2.55 \mathrm{~m}$. As seen from Table 1, the response of the craft to the step-like wind gust of $1 \mathrm{~m} / \mathrm{sec}$ incrreases when the $\mathrm{CG}$ is shifted towards the leading edge (CG of 1.637 and $1.887 \mathrm{~m}$ ). When the $\mathrm{CG}$ position between the aerodynamic centers, i.e., $2.10 \mathrm{~m}<\mathrm{CG}$ position $<$ $2.55 \mathrm{~m}$, the response becomes smoother. This is in agreement with recommendation (5). The explanation of this fact is straightforward. When $C G$ is shifted towards the aerodynamic center on pitch, the value $X_{\vartheta}$ becomes smaller. Therefore, as follows from Eq. (3), the oscillation magnitude of the flight altitude decreases as well.

The results of simulation of the response to harmonic wind gusts are presented in the Fig. 9. The smallest response corresponds to the $\mathrm{CG}$ position chosen from the rule $(5)$. In this case WIG craft can fly without pitch-up tendency and touching the water surface at wind speeds up to $13.5 \mathrm{~m} / \mathrm{sec}$.

The response of WIG craft to the waves is much milder than that to the wind gusts. ${ }^{5}$ The wind gust limitation is more important than a limitation due to the wave influence for the small WIG craft flying in rough sea conditions.

\section{Safety of manoeuvring}

A WIG craft can avoid collisions with other objects by fulfilling either the horizontal manoeuvre (banked turning) (Fig. 10a) or jumping in the vertical plane (Fig. 10b). The horizontal manoeuvre is much safer than the vertical one due to the risk of the pitch-up instability in the second case. The turning radius is approximately a few hundreds meters for small craft $(300 \mathrm{~m}$ for the Hovering 2VT) and a few kilometres for large machines. When turning, the WIG craft experiences an additional drag caused by the drift, roll angles and deflection of control elements. To overcome possible touchdown in a turning manoeuvre, a special strategy was developed by the Alekseev Bureau and independently by Airfoil Development. The active control system keeps the distance between the wing tip and the ground surface constant. Therefore, before the turning manoeuvre, a WIG craft has to attain a larger flight altitude by increasing thrust and actuating ailerons (Fig. 11).

The jumping manoeuvre can be performed to avoid colliding with low obstacles, when they are discovered at a short distance ahead of a WIG craft. For instance, for the WIG craft Hydrowing VTOI the minimum possible distance to avoid an obstacle of characteristic size of 1 meter by jumping is three times less than that needed for turning manoeuvre.

\section{Safety of transition modes}

The take-off regime has proved to be the most dangerous phase of the WIG motion. To overcome the hump drag, even the best skimming surfaces require approximately 2.3 times more power than that needed for ground effect cruising. Depending on the design, the mutual disposition of the drag and thrust curves can be of two types (Fig. 12). In the first case (Drag 1) the thrust is counterbalanced by the drag without any pilot actions at the point $\mathrm{A}$. In the second case (Drag 2), at a detachment moment a pilot has to reduce the thrust from a full throttle to a value providing a stable flying at a given altitude (point $\mathrm{C}$ ). The second case is typical for small WIG craft; and safe performance of the takeoff is a real problem for inexperienced pilots. At the moment of detachment the contact with water disappears, and at the same time hydrodynamic damping stops providing the stabilized action on WIG motion. If a pilot did not react properly, then the over thrust appears that leads to the sudden increase of the flight altitude, to the loss of the longitudinal stability, to the raise of the pitch angle, and finally to the stall effect. This is a typical scenario of most crashes happened with WIG craft. The risk of the pitch-up tendency during the take-off can be reduced by proper positioning the center of gravity and by correct piloting strategy.

Two different strategies for the engine control aimed at avoiding critical unstable behaviour of WIG craft the take-off mode have been proposed and investigated for the second type of Drag-Thrust diagram. ${ }^{5}$ The simple recommendation is the following: a pilot should provide a steep reduction of the thrust down to the value $T_{c}$ needed for the cruise regime immediately after the detachment moment, which is easily indicated (Curve O-A-B-C). The value $T_{c}$ can be estimated a priori on the base of calculations or measurements.

A special recommendation is developed for the case when a pilot fails to perform the take-off properly, and the WIG motion becomes critical. The early indication of a critical behaviour is the excessive speed of the motion (neither the pitch angle nor the height of flight). According to our numerical simulation, a pilot should turn the engine off, if the speed of motion is more than a given cruise speed of the WIG, and turn it on again when the speed of motion becomes less than or equal to the speed at the detachment moment.

\section{Conclusions}

The presented mathematical tool is capable of modeling aerodynamics of a WIG system, including a ground effect, hydrodynamics of a stepped planing hull, and simulation of WIG motion. The extensive experience of using this model proves that the most important and necessary features of the WIG aero- and 
hydrodynamics are taken into account properly. The proposed complex mathematical model for WIG motion is thoroughly tested for different characteristics in planing and flight modes. Simulation results illustrate the ability of the model to investigate WIG motion in normal and emergent conditions.

\section{References}

[a] N.I. Belavin. Ekranoplans. Sudostroenie, Leningrad, 1977. (in Russian)

[b] E.A. Aframeev. Conceptual bases of WIG craft building: ideas, reality and outlooks. RTO AVT Symposium on Fluid Dynamics Problems of Vehicles Operating Near or in the Air-Sea Interface, Amsterdam, 1998.

[c] N. Tomita, A.V. Nebylov, V.V. Sokolov, D. Tsumrumaru, T. Saotome and Y. Ohkami. Feasibility study of a rocket-powered HTHL-SSTO with Ekranoplane as Takeoff Assist. AIAA 96$4517,1996$.

[d] W. Cole. The Pelican: a big bird for the long haul. Boeing Frontiers Online, Volume 1, Issue 05, 2002.

http:/www.boeing.com/news/frontiers/index.html

[e] K.V. Rozhdestvensky. Aerodynamics of a Lifting System in Extreme Ground Effect. Sringer-Verlag, Berlin, 2000.

[1] A.I. Maskalik, K.V. Rozhdestvensky and D.N. Synitsin. A view of the present state of research in aero- and hydrodynamics of ekranoplans. RTO AVT Symposium on Fluid Dynamics Problems of Vehicles Operating Near or in the Air-Sea Interface, Amsterdam, 1998.

[2] WIG Page, http://www.se-technology.com/

[3] H. Fischer and K. Matjasic. From Airfisch to Hoverwing. Workshop WISE up to ekranoplan GEMs, The University New South Wales, 69-89, 1998.

[4] N.V. Kornev and A.E. Taranov, 1998. AutoWing 2.4, http://www.cl.spb.ru/taranov/Index.htm.

[5] K. Benedikt, N.V. Kornev, M. Meyer and J. Ebert. Complex mathematical model of the WIG motion including the take-off mode, Ocean Engineering, Vol. 29, 315-357, 2001 .
[6] N.V. Kornev and V.K. Treshkov. Numerical investigation of nonlinear unsteady aerodynamics of the WIG Vehicle. Intersociety Conference on High Performance Marine Vehicles, Arlington, VA, USA, ws38-ws48, 1992.

[7] V.I. Zhukov. Peculiarities of the WIG's aerodynamics, dynamics and stability. TSAGI Press, 1998. (in Russian).

[8] V.I. Zhukov, private communication, 2000.

[9] R. Brockhaus. Flugregelung. Springer, 1994. 


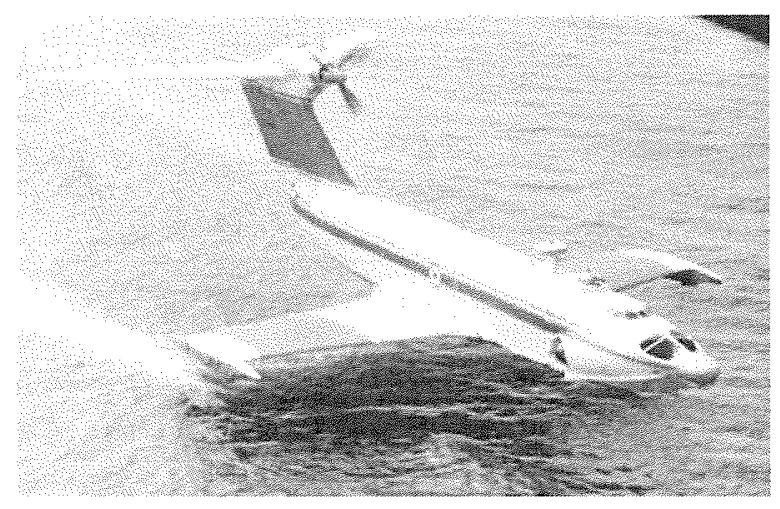

Fig. 1: Ekranoplan Orlyonok.

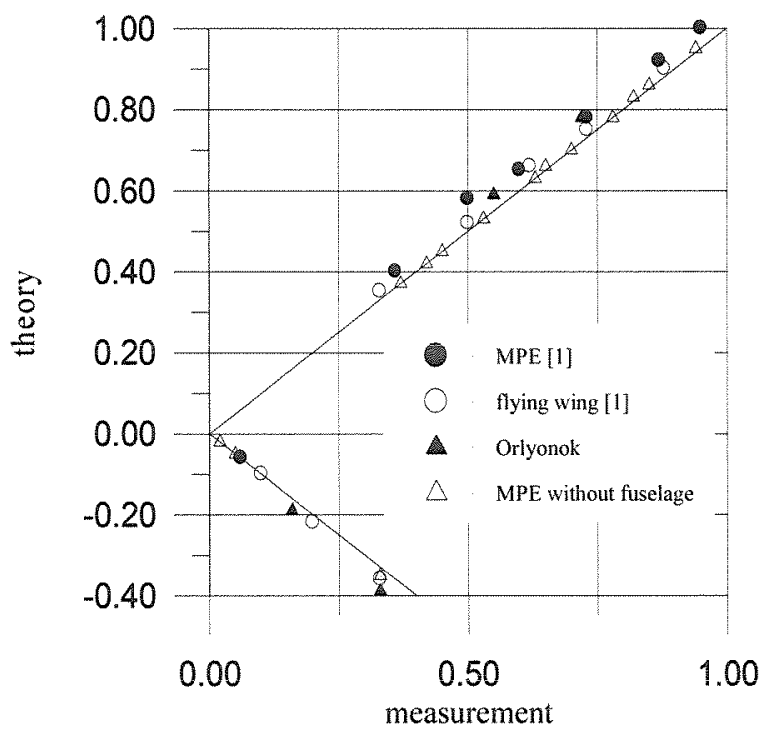

Fig. 2: Lift and moment coefficients. Comparison with measurements for different WIG craft.

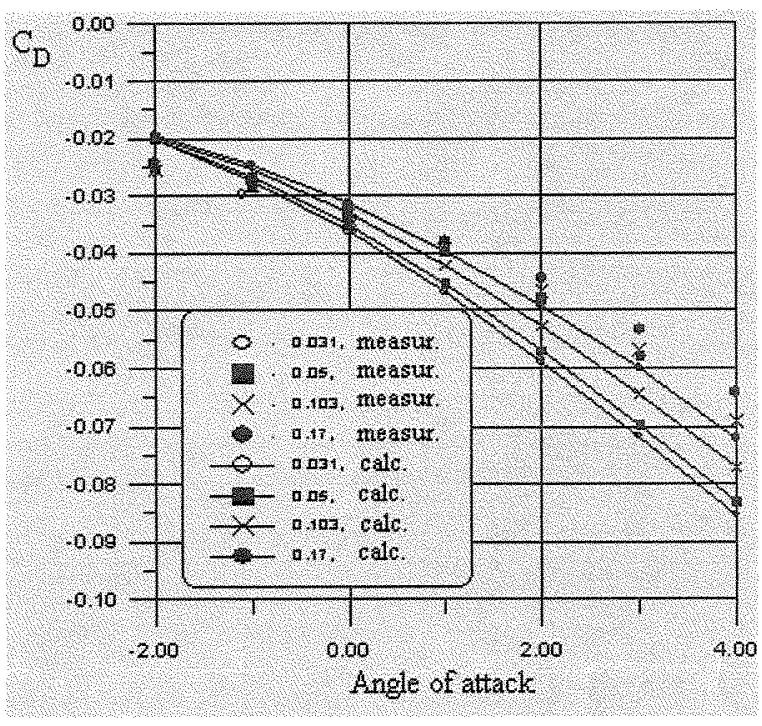

Fig. 3: Drag coefficient versus the angle of attack for different altitudes; WIG Hydrowing [6].

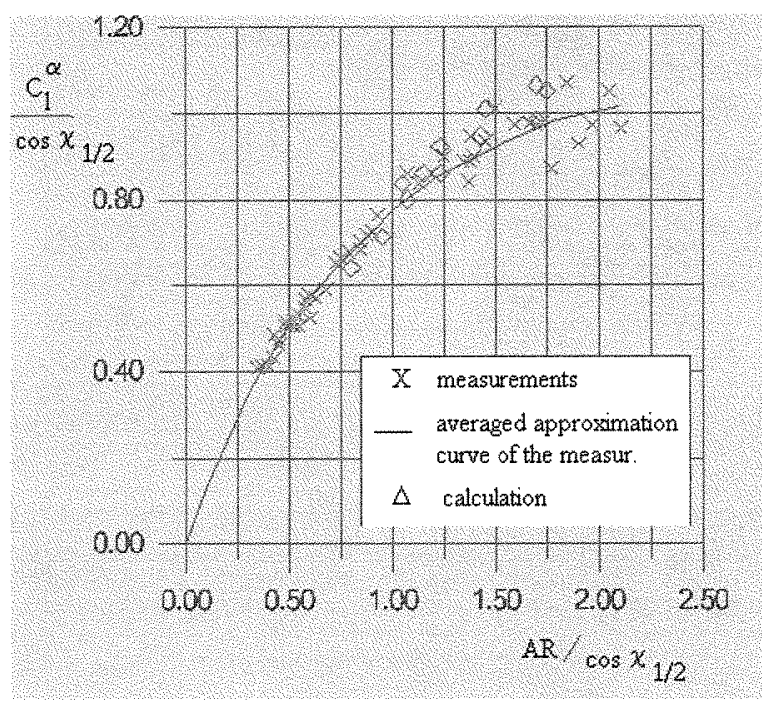

Fig. 4: Slope of the lift coefficient versus aspect ratio of the planning surface. 


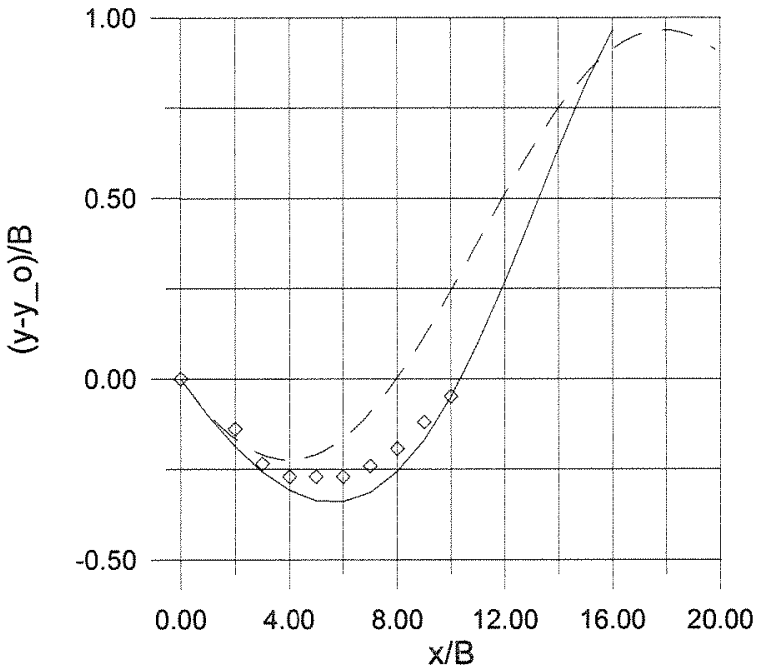

Fig. 5: The shape of the wave wake in the symmetry plane behind a flat plate at pitch angle of $6^{\circ}$.

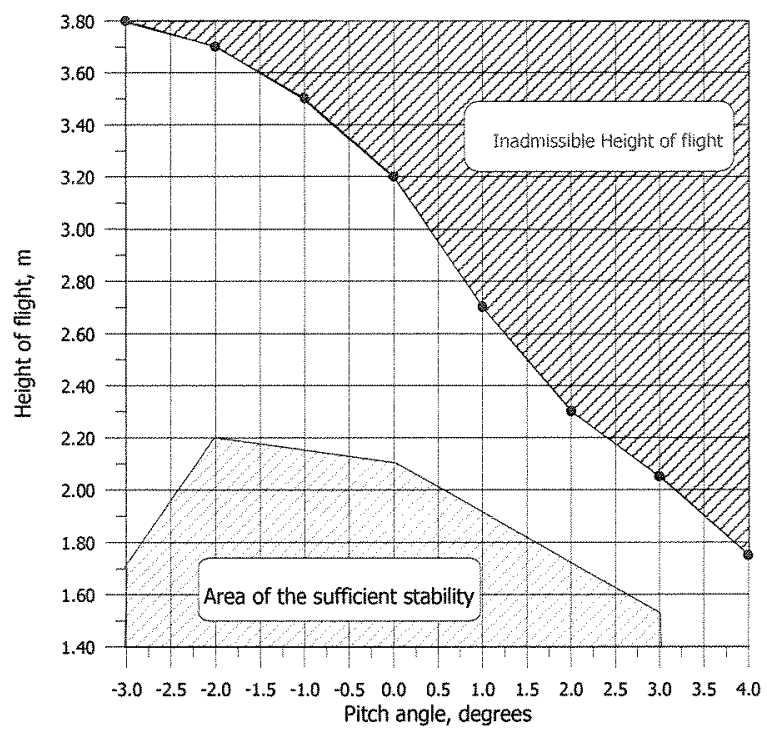

Fig. 6: Areas of the stability for the WIG craft "Seajet S.d.51". The height of flight is measured from the trailing edge of the wing in the tip section. The reference point for the criterion of the static stability was located at the distance $3.662 \mathrm{~m}$ from the trailing edge.

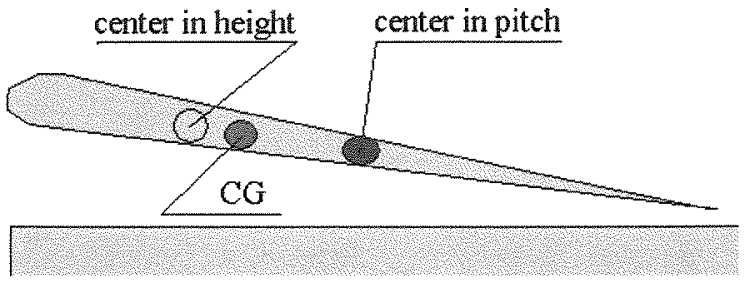

Fig. 7: Favourable mutual positions of the center of gravity (SP), the aerodynamic center on altitude (NA) and the aerodynamic center on pitch (NP).

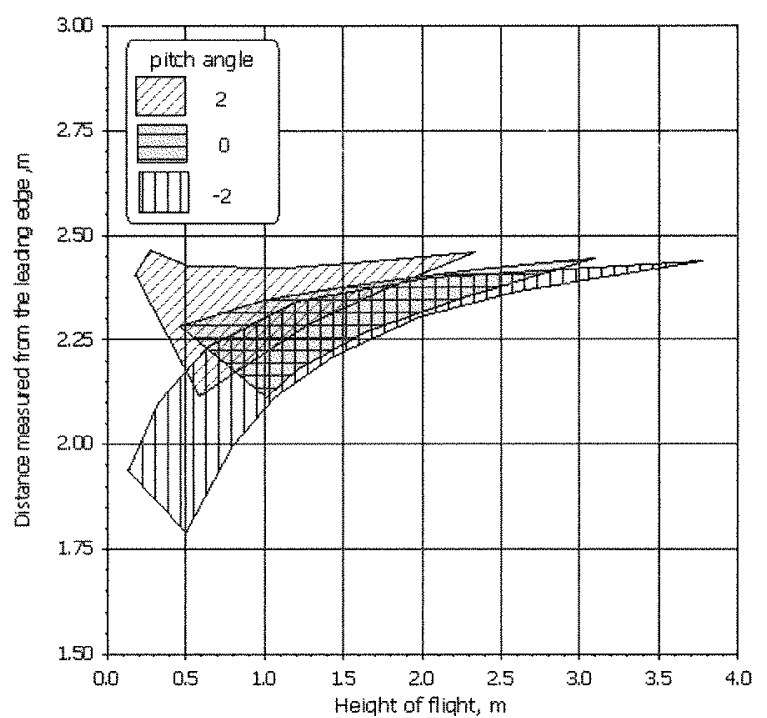

Fig. 8: Favourable position of the center of gravity.

\begin{tabular}{|l|l|l|}
\hline $\begin{array}{l}\text { CG position, } \\
\mathrm{m}\end{array}$ & $\begin{array}{l}\text { Max amplitude } \\
\text { of vertical } \\
\text { oscillations, } \mathrm{m}\end{array}$ & $\begin{array}{l}\text { Max amplitude } \\
\text { of pitch } \\
\text { oscillations, deg }\end{array}$ \\
\hline 1.637 & 1.44 & 0.79 \\
\hline 1.887 & 1.09 & 0.49 \\
\hline 2.187 & 0.735 & 0.21 \\
\hline 2.437 & 0.40 & 0.29 \\
\hline
\end{tabular}

Table 1: Parameters of oscillations caused by the step-like head wind gust $1 \mathrm{~m} / \mathrm{sec}$. 


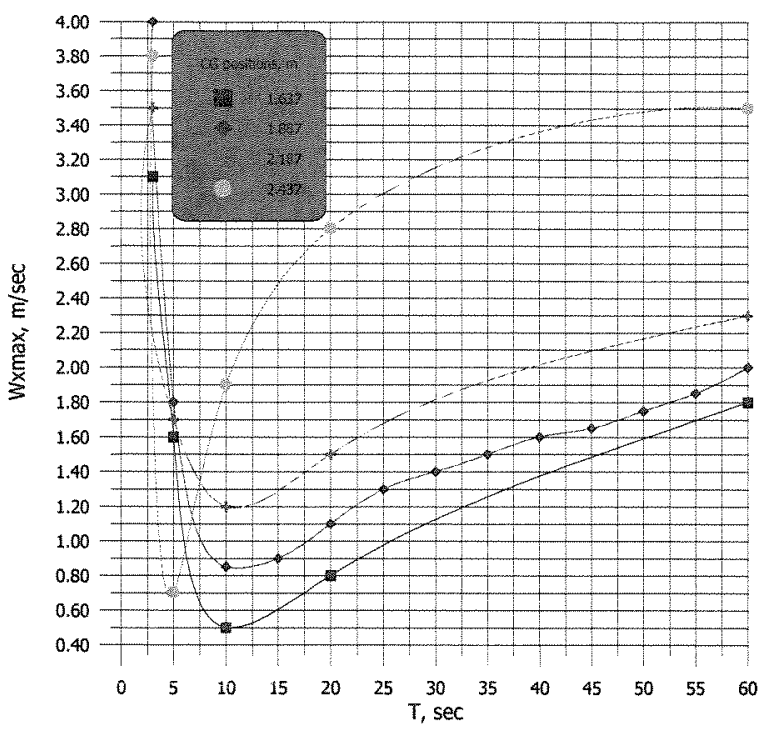

Fig. 9: Maximum permissible wind gust versus the period T.

a)

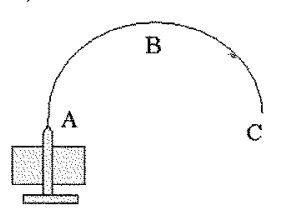

b)

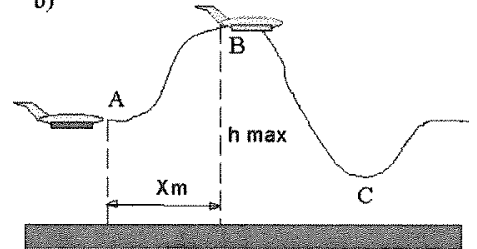

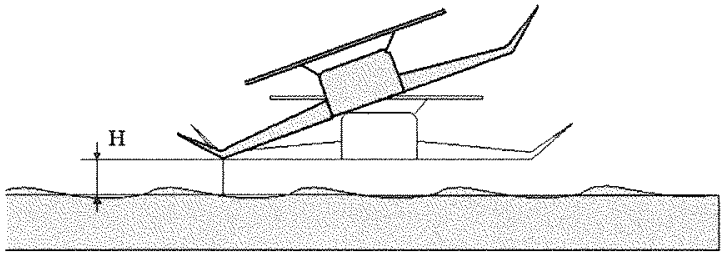

Fig. 11: Technique of turning.

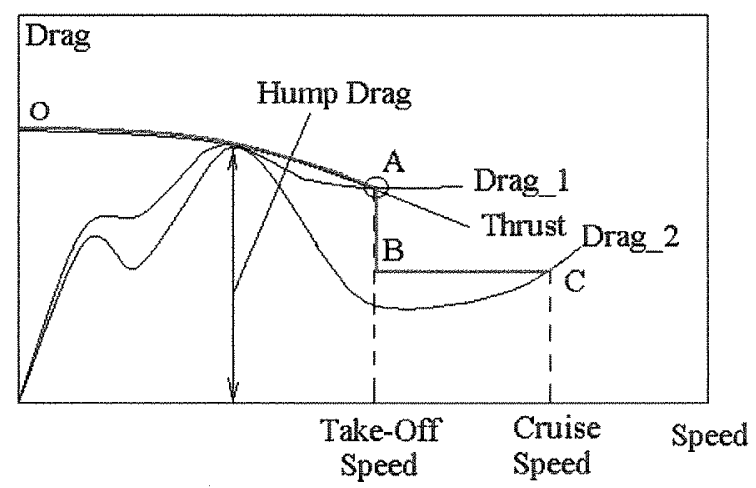

Fig. 12: Typical disposition of the Drag and Thrust curves.

Fig. 10: Two types of manoeuvres to avoid colliding with obstacles: (a) horizontal manoeuvre; (b) vertical jumping. 